\title{
ATLAS studies of diffraction, soft particle production and double parton scattering
}

\section{Timothy A. D. Martin*}

On behalf of the ATLAS collaboration

University of Birmingham

E-mail: tim.martin@cern.ch

\begin{abstract}
Soft and semi-hard QCD studies made with ATLAS at the LHC are presented. These include measurement of the inelastic and diffractive cross sections, mean forward energy flow, forwardbackward and azimuthal correlations, azimuthal ordering of hadron production, event shapes, and identified strange particle production. Distributions sensitive to the underlying event are measured in a variety of hard processes. In addition, an explicit study of double-parton scattering using $\mathrm{W}+$ dijet events is presented, along with a measure of the effective cross section. Many of these measurements are used to develop and tune models for soft particle production. An overview of these results is given.
\end{abstract}

$36^{\text {th }}$ International Conference on High Energy Physics

4-11 July 2012

Melbourne, Australia

${ }^{*}$ Speaker. 


\section{Introduction}

Recent studies by ATLAS of minimum bias events and the underlying event at the LHC are briefly outlined below. These are valuable data for studying inter-particle correlations, and for improving our modelling of the physics of soft and semi-hard hadronic interactions.

Events were typically triggered via the Minimum Bias Trigger Scintillators (MBTS) which, during early running, were highly sensitive to the passage of charged particles and occupy the interval ${ }^{1} 2.09<|\eta|<3.84$ [1]. Further selection was available in the High Level Trigger (HLT) with the possibility to discriminate based on the number of Inner Detector (ID) hit or reconstructed tracks. The ATLAS detector is described in detail elsewhere [2]. All of the presented analyses use the inner tracking detectors which span the range $|\eta|<2.5$ and reconstruct charged particles with $p_{\mathrm{T}} \geq 100 \mathrm{MeV}$. Some analyses additionally utilise the calorimeter systems which hermetically cover $|\eta|<4.9$.

ATLAS minimum bias data are fully corrected for detector effects using a variety of statistical treatments and fully reconstructed Monte Carlo (MC) simulations.

\section{Inelastic Cross Sections Measurements}

The inelastic $p p$ cross section subdivides, somewhat ambiguously, into diffractive and nondiffractive (ND) components. The larger non-diffractive cross section originates primarily through the exchange of a colour-octet and is modelled in the Pythia MC via lowest-order $2 \rightarrow 2$ scattering with phenomenological renormalisation of divergences at small $p_{\mathrm{T}}$. Diffractive interactions are mediated via the exchange of a colour singlet, the lack of colour flow suppresses soft gluon radiation between the protons and results in events containing large pseudorapidity gaps, devoid of final state particles. The size of the pseudorapidity gap is kinematically linked to the invariant mass of the diffractive system $\left(M_{X}\right)$. Through the use of the phenomenology of Regge theory, the leading form factor of the diffractive cross section goes like $1 / M_{X}^{2}$ which leads to an approximately flat cross section in pseudorapidity gap size. The variable $\Delta \eta^{F}$ is defined as the largest continuous interval in $\eta$ which stretches inward from either edge of the ATLAS detector at $|\eta|=4.9$ and which contains no particles with $p_{\mathrm{T}}$ above a fixed cut off. The variable is studied in the range $0<\Delta \eta^{F}<8$ for $200<p_{\mathrm{T}}^{\text {cut }}<800 \mathrm{MeV}$ [3]. The distribution is truncated at $\Delta \eta^{F}=8$ due to falling trigger efficiency and the range of values for $p_{\mathrm{T}}^{\text {cut }}$ allow for the study of pseudorapidity gaps in hadronisation. The data are presented at $p_{\mathrm{T}}^{\text {cut }}=200 \mathrm{MeV}$ in Fig. 1(a), the exponentially falling cross section at small gap size is due to hadronisation - predominantly from ND events. Diffractive events are isolated at large gap sizes, the slow rise in the cross section around $\Delta \eta^{F}=7$ is shown to be compatible with a supercritical Pomeron as favoured by Donnachie and Landshoff in fitting the slow rise of the total cross section with $\sqrt{s}$ [4].

The total inelastic cross section is measured in [5]. Due to the location of the MBTS trigger, low-mass diffractive interactions are not present in the minimum bias data sample and ATLAS

\footnotetext{
${ }^{1}$ In the ATLAS coordinate system, the $z$-axis points in the direction of the anti-clockwise beam viewed from above. Polar angles $\theta$ and transverse momenta $p_{\mathrm{T}}$ are measured with respect to this axis. The pseudorapidity $\eta=-\ln \tan (\theta / 2)$ is a good approximation to the rapidity of a particle whose mass is negligible compared with its energy and is used here, relative to the nominal $z=0$ point at the centre of the apparatus, to describe regions of the detector.
} 


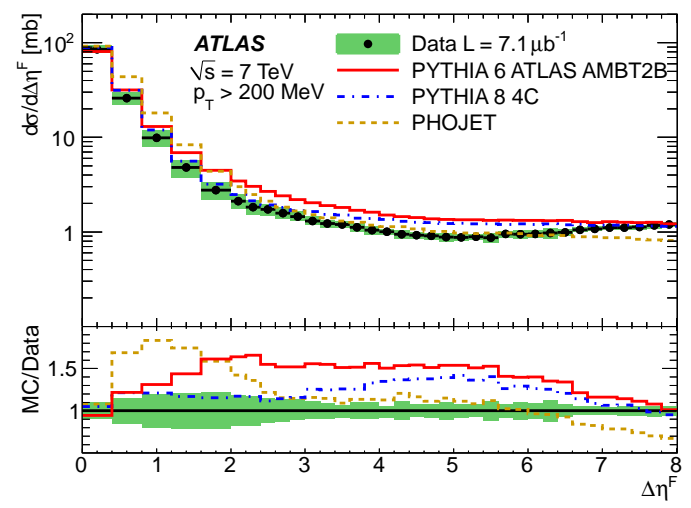

(a)

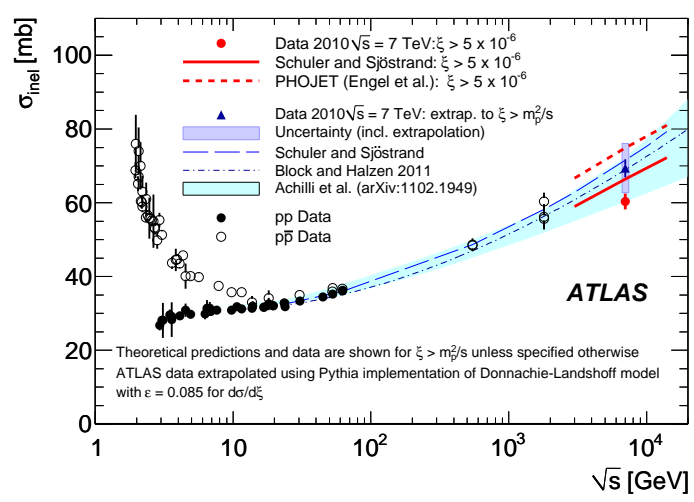

(b)

Figure 1: (a) Inelastic cross section differential in $\Delta \eta^{F}$ [3] and (b) total inelastic cross section [5].

measures the cross section for events with $\xi>5 \times 10^{-6}$ (where $\xi=M_{X}^{2} / s$ ) to be $\sigma_{\text {inela }}(\xi>5 \times$ $\left.10^{-6}\right)=60.30 \pm 0.05$ (stat) \pm 0.50 (syst) \pm 2.1 (lumi) mb. The data in Fig. 1(b) are subsequently extrapolated to all values of $\xi$ using various models of diffraction which yields a value for the total inelastic cross section compatible with the precision measurement of TOTEM [6].

\section{Particle Correlations}

The latest analysis on the Underlying Event (UE) presented in [7] is based on charged particle track-jets. In events containing a hard-scale scatter, the UE is formed from the additional interactions between the protons which do not originate from the hard scatter. The anti- $k_{t}$ algorithm [8] is used to reconstruct these track-jets with radius parameter $0.2<R<1.0$. The data provide additional detail in the transition from studying the UE in leading-track data ${ }^{2}$ to calorimeter jets data [9]. The charged particle multiplicity, sum transverse momentum $\left(\Sigma\left|p_{\mathrm{T}}\right|\right)$ and mean transverse momentum $\left(\bar{p}_{\mathrm{T}}\right)$ are studied at different $R$ values for track-jets in the range $4<p_{\mathrm{T}}^{\text {jet }}<100 \mathrm{GeV}$ in the transverse and away regions. The transverse region is defined as the two sixths of the azimuth $\frac{\pi}{3}<|\Delta \phi|<\frac{2 \pi}{3}$ where $\Delta \phi$ is the distance in $\phi$ from the leading track-jet. This region is the most sensitive to the UE and is used to study the correlations between the hard scatter and any additional interactions between the proton remnants. The away region is the third of the azimuth at $|\Delta \phi|>\frac{2 \pi}{3}$ which typically contains the hadronic recoil. Pythia 6 tunes Z1, AUET2B and Perugia2011 are shown to perform the best. In Fig. 2(a), the ratio of the average summed $p_{\mathrm{T}}$ between $R=0.2$, $R=0.6$ and $R=1.0, R=0.6$ is compared to $\mathrm{Z} 1$. Good agreement is observed for this challenging variable.

In [10], the amount of forward-backward (in $\eta$ ) correlation is measured for charged particle multiplicity and $\Sigma\left|p_{\mathrm{T}}\right|$. Five equally sized $\eta$ regions are defined between $\eta=0$ and $\eta=2.5$. Each of these $\eta$ regions in the forward direction $(+z)$ is compared to its symmetric counterpart in the backward direction $(-z)$ in events which contain at least two charged particles with $p_{\mathrm{T}}>100 \mathrm{MeV}$. The discriminating variable for multiplicity is the normalised covariance between the forward and

\footnotetext{
${ }^{2}$ which may be considered analogous to the anti- $k_{t} R=0$ case.
} 


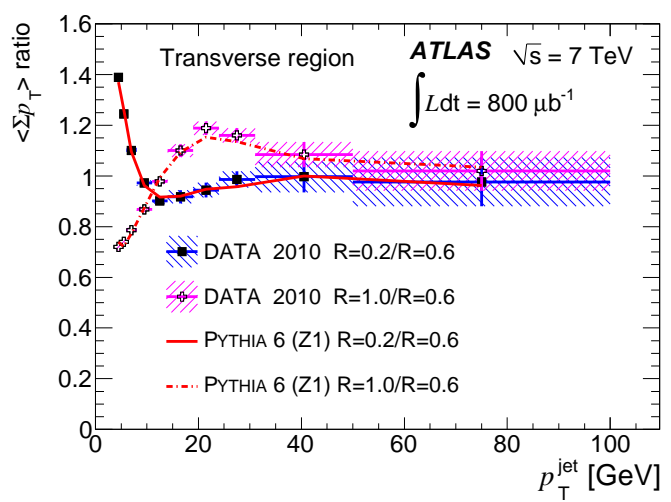

(a)

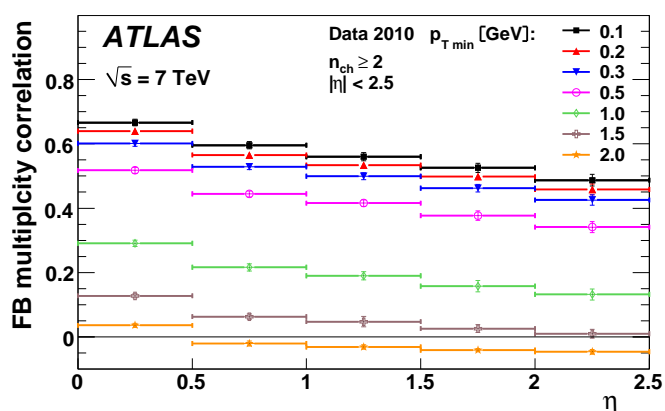

(b)

Figure 2: (a) Ratio of average sum $p_{\mathrm{T}}$ in the UE transverse region at different $R$ values [7]. (b) Strength of forward-backward charged particle multiplicity correlation as a function of minimum particle $p_{\mathrm{T}}[10]$.

background regions, relative to the mean values of each, $\rho_{f b}^{n}=\frac{\sum x_{f}^{n} x_{b}^{n}}{N \sigma_{f}^{n} \sigma_{b}^{n}}$. Here the sum is over all events, $N$ is the number of events, $x^{n}$ is the deviation of the per-event multiplicity from the mean value, $\sigma^{n}$ is the standard deviation of the multiplicity distribution about its mean and $f$ and $b$ denote the forward or backward regions. With charged particle multiplicity replaced with summed absolute $p_{\mathrm{T}}$, the $\Sigma\left|p_{\mathrm{T}}\right|$ asymmetry is obtained. The strength of both correlations is shown to fall with increased separation and differ by up to $15 \%$ when compared to standard $\mathrm{MC}$ tunes at $7 \mathrm{TeV}$ and over $20 \%$ for some cases at $900 \mathrm{GeV}$. In Fig. 2(b) the multiplicity correlation is plotted for the five $\eta$ regions as a function of the minimum $p_{\mathrm{T}}$ required for a particle to be included in the sample. The correlation is observed to fall with increased minimum $p_{\mathrm{T}}$, even showing negative correlation at $p_{\mathrm{T}}^{\min }=2 \mathrm{GeV}$, hinting at the onset of jet structure formation.

The generalised two particle correlation is additionally measured in [11] using a multiplicityindependent correlation function $R(\Delta \eta, \Delta \phi)$ over all pairs of particles in each event. The correlation distribution is divided by the background distribution formed by combining pairs of particles between events and the data are unfolded in a model independent manner via the novel HBOM approach [12]. Presented in Fig. 3(a) is a one dimensional projection of $R(\Delta \eta)$ integrated over $0<\Delta \phi<\pi$ for events containing two or more charged particles with $p_{\mathrm{T}}>100 \mathrm{MeV}$. None of the considered models are able to provide a good description of the strength of the correlations over the full phase space.

\section{Transverse Energy Density and Event Shapes}

The transverse energy density, $\Sigma E_{\mathrm{T}}$, of charged(neutral) particles with $p_{\mathrm{T}}>500(200) \mathrm{MeV}$ is measured in bins of $\eta$ up to $|\eta|<4.8$ for a minimum bias and di-jet data sample [13]. The minimum bias sample studies the whole azimuth for events with at least two reconstructed charged particles with $p_{\mathrm{T}}>250 \mathrm{MeV}$ in the ID. For the di-jet sample, at least two di-jets must be reconstructed with the anti- $k_{t}$ algorithm $(R=0.4)$ which both satisfy $E_{\mathrm{T}}^{\text {jet }}>20 \mathrm{GeV}$ and $\left|\eta^{\text {jet }}\right|<2.5$. The dijet system must in addition be well balanced, with $E_{\mathrm{T}}^{\mathrm{jet} 1} / E_{\mathrm{T}}^{\mathrm{jet} 2}>0.5$ and $\left|\phi^{\mathrm{jet} 1}-\phi^{\mathrm{jet} 2}\right|>2.5$. The 


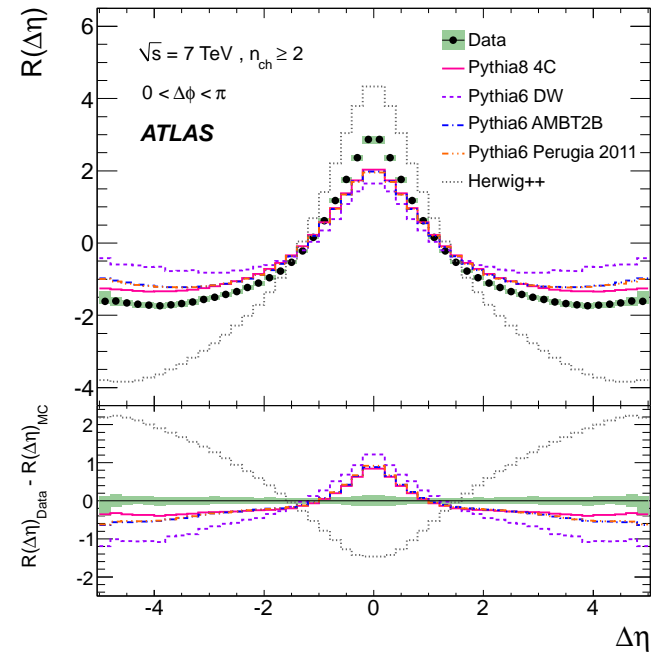

(a)

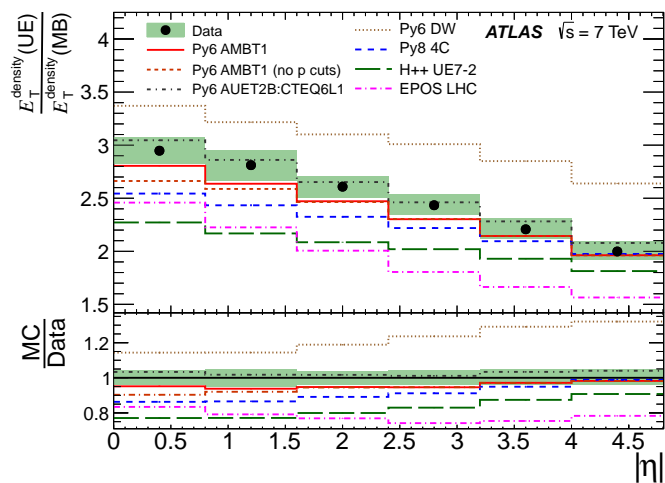

(b)

Figure 3: (a) Two particle correlation function $R(\Delta \eta)$ integrated over $0<\Delta \phi<\pi$ [11]. (b) Ratio of $E_{\mathrm{T}}$ density in the di-jet transverse region divided by the minimum bias sample [13].

measurement for this selection is restricted to the transverse region of the azimuth with respect to the di-jet system to probe energy flow in the underlying event.

In the forward region, most $\mathrm{MC}$ models are shown to underestimate the average energy density by $20-30 \%$. The di-jet distribution is best modelled by the DW tune of Pythia 6 while the minimum bias sample is best modelled by EPOS, which is a generator used more commonly for cosmic ray air-shower simulation. In addition, changing the gluon PDF from CTEQ6L1 to MSTW2008 LO enhances the forward energy density to be more in line with data. The ratio between the $E_{\mathrm{T}}$ density in di-jet (transverse) and minimum bias events is shown in Fig. 3(b) with the former possessing a larger $E_{\mathrm{T}}$ density in the vicinity of the hard scatter.

In [14], three event shape quantities; the transverse thrust, thrust minor and transverse sphericity [15] are studied in the range $|\eta|<2.5$ for events containing at least six tracks with $p_{\mathrm{T}}>0.5 \mathrm{GeV}$. These variables are defined such that they are maximal for more spherical topologies. The data and MC are compared for events which satisfy $p_{\mathrm{T}}^{\text {lead }}=0.5,2.5,5.0 \mathrm{GeV}$ where $p_{\mathrm{T}}^{\text {lead }}$ is the highest $p_{\mathrm{T}}$ track in the event. The shift from a greater spherical symmetry in events at low $p_{\mathrm{T}}^{\text {lead }}$ (Fig. 4(a)) to more pencil-like topologies at larger $p_{\mathrm{T}}^{\text {lead }}$ (Fig. 4(b)) is clearly observed. None of the MC models considered predict the fraction of spherical events, with tunes of UE data outperforming those based on minimum bias data. The averages of the event shape quantities are additionally studied as a function of the charged particle multiplicity and $\Sigma\left|p_{\mathrm{T}}\right|$, both of which saturate towards a more spherical topology at the upper measured values of $N_{\mathrm{ch}}=70$ and $\Sigma\left|p_{\mathrm{T}}\right|=100 \mathrm{GeV}$.

\section{Double Parton Interactions}

The effective cross section for double parton scattering in $p p$ events is extracted from a sample of $W \rightarrow l v+j j$ events where $l=e, \mu$ [16]. Under the assumption that the two scatters are independent, the di-jet system in DPI events is expected to be back-to-back in $\phi$. The $p_{\mathrm{T}}$ 


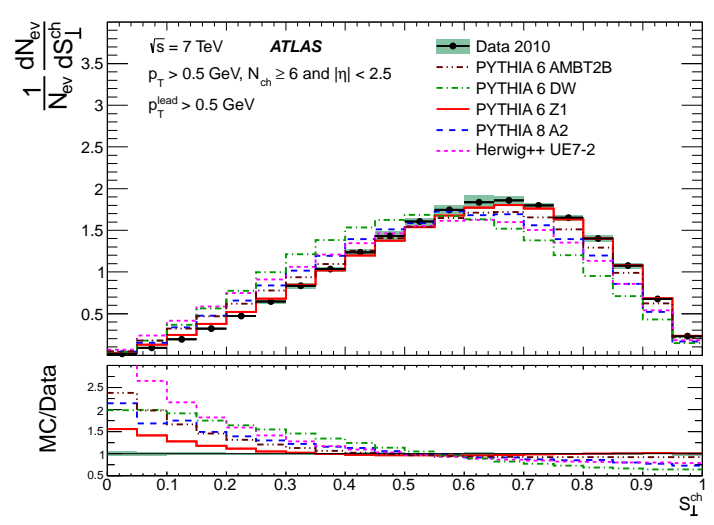

(a)

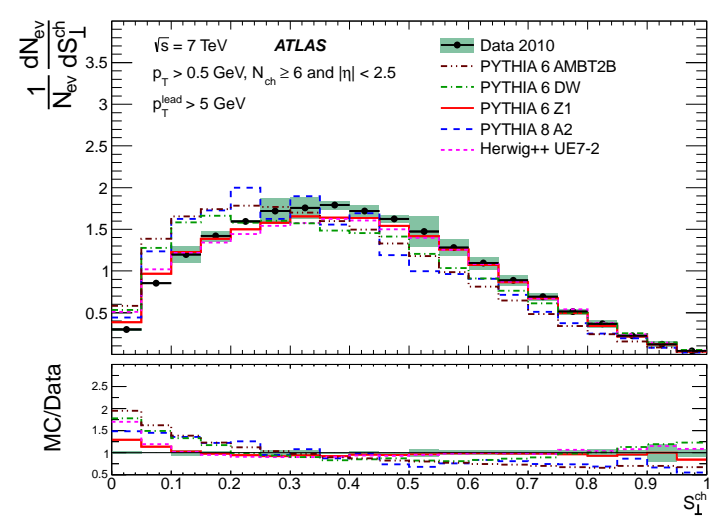

(b)

Figure 4: Transverse sphericity for $p_{\mathrm{T}}^{\text {lead }}=0.5 \mathrm{GeV}$ (a) and $p_{\mathrm{T}}^{\text {lead }}=5.0 \mathrm{GeV}$ (b) [14].

imbalance is quantified with the variables $\Delta_{\text {jets }}=\left|\vec{p}_{\mathrm{T} 1}+\vec{p}_{\mathrm{T} 2}\right|$ and $\Delta_{\text {jets }}^{n}=\left|\vec{p}_{\mathrm{T} 1}+\vec{p}_{\mathrm{T} 2}\right| /\left|\vec{p}_{\mathrm{T} 1}\right|+\left|\vec{p}_{\mathrm{T} 2}\right|$ where 1 and 2 identify the jets. The fraction of DPI is extracted from the distributions via templated fits to the data. The two templates used are MC $W \rightarrow l v$ samples (using Sherpa or Alpgen+Herwig+Jimmy) and a di-jet data sample, backgrounds from multi-jet, $W \rightarrow \tau \nu, t \bar{t}$ and $Z \rightarrow l l$ are also included. The templated extraction of the DPI fraction $f_{\text {DPI }}=0.16 \pm 0.01$ (stat) \pm 0.03 (sys) is subsequently used to calculate the effective cross section $\sigma_{\mathrm{DPI}}^{\text {eff }}(7 \mathrm{TeV})=11 \pm 1$ (stat $)_{-2}^{+3}($ sys $) \mathrm{mb}$.

\section{References}

[1] T.A. Martin, JINST 5(12), C12051 (2010). DOI 10.1088/1748-0221/5/12/C12051

[2] ATLAS Collaboration, JINST 3(08), S08003 (2008). DOI 10.1088/1748-0221/3/08/S08003

[3] ATLAS Collaboration, Eur.Phys.J. C72, 1926 (2012). DOI 10.1140/epjc/s10052-012-1926-0

[4] P. Landshoff, arXiv:hep-ph/9410250 (1994)

[5] ATLAS Collaboration, Nature Commun. 2, 463 (2011). DOI 10.1038/ncomms 1472

[6] TOTEM Collaboration, Europhys. Lett. 96, 21002 (2011). DOI 10.1209/0295-5075/96/21002

[7] ATLAS Collaboration, arXiv:hep-ex/1208.0563 (2012)

[8] M. Cacciari, G.P. Salam, G. Soyez, JHEP 0804, 063 (2008). DOI 10.1088/1126-6708/2008/04/063

[9] ATLAS Collaboration, Eur.Phys.J. C71, 1636 (2011). DOI 10.1140/epjc/s10052-011-1636-z

[10] ATLAS Collaboration, JHEP 1207, 019 (2012). DOI 10.1007/JHEP07(2012)019

[11] ATLAS Collaboration, JHEP 1205, 157 (2012). DOI 10.1007/JHEP05(2012)157

[12] J.W. Monk, C. Oropeza-Barrera, arXiv:hep-ex/1111.4896 (2011)

[13] ATLAS Collaboration, arXiv:hep-ex/1208.6256 (2012)

[14] ATLAS Collaboration, arXiv:hep-ex/1207.6915 (2012)

[15] A. Banfi, G.P. Salam, G. Zanderighi, JHEP 1006, 038 (2010). DOI 10.1007/JHEP06(2010)038

[16] ATLAS Collaboration, ATLAS-CONF-2011-160 (2011) 